\title{
Experimental electronic stopping cross section of transition metals for light ions: Systematics around the stopping maximum
}

\author{
M. V. Moro $\odot,{ }^{1, *}$ P. Bauer, ${ }^{2}$ and D. Primetzhofer $\circledast^{1}$ \\ ${ }^{1}$ Department of Physics and Astronomy, Uppsala University, Box 516, 75120 Uppsala, Sweden \\ ${ }^{2}$ Atomic Physics and Surface Science, Johannes Kepler University, A-4040 Linz, Austria
}

(Received 27 May 2020; accepted 14 July 2020; published 12 August 2020)

\begin{abstract}
Electronic stopping cross sections of different transition metals $(\mathrm{Nb}, \mathrm{Pd}$, Ta, and $\mathrm{Pt}$ ) for light ions have been experimentally determined in a wide energy range. We performed relative measurements using different backscattering geometries for protons (from 50 to $5000 \mathrm{keV}$ ) and helium (from 80 to $10000 \mathrm{keV}$ ). Data are compared to values from the literature, as well as to the widely used semiempirical (SRIM) and modeling (DPASS) approaches. The magnitude and energy dependence of the deduced stopping power at energies around the Bragg peak, as well as the different trends observed within individual periods, are analyzed with respect to target atomic number and electronic structure. We also compare the observed magnitude of electronic stopping to several different theoretical approaches.
\end{abstract}

DOI: 10.1103/PhysRevA.102.022808

\section{INTRODUCTION}

When an energetic ion penetrates matter, it loses kinetic energy along its path, due to collisions with target nuclei and electrons. The quantity that describes the resulting average energy loss per unit path length is commonly denoted as the stopping power $S=d E / d x$ of the material for a specific ion, which can, based on the nature of the interaction, be separated into a nuclear and an electronic component [1,2]. The stopping power of matter for ions has been under investigation for over a century [3] and has become a decisive quantity in ion beam analysis [4,5] and fusion research [6,7], as well as medical applications [8,9]. For many of these applications, it is convenient to normalize $S$ by the atomic density $n$ of the material to get rid of the trivial dependence on the material density. The resulting quantity is commonly referred to as stopping cross section, $\varepsilon=S / n$.

Due to the vast number of ion-material combinations, a theoretical prediction of $\varepsilon$ is highly desirable. At high ion energies $\left(v \gg v_{F}\right)$, i.e., when the ion velocity $v$ is high compared to the Fermi velocity $v_{F}$ of the target electrons $\left(v \leqslant v_{F}\right)$, the ion can be considered only a weak perturbation of the target electronic system, and the process is commonly described by first-order theoretical approaches [10,11]. At intermediate and low ion energies, i.e., around and below a pronounced maximum in $\varepsilon$, the theoretical framework becomes more complex, as the electronic energy loss is predominantly due

\footnotetext{
*marcos.moro@physics.uu.se
}

Published by the American Physical Society under the terms of the Creative Commons Attribution 4.0 International license. Further distribution of this work must maintain attribution to the author(s) and the published article's title, journal citation, and DOI. Funded by Bibsam. to interactions with the valence electrons of the target. At sufficiently low energies $\left(v<v_{F}\right)$, the stopping process can be finally described as the interaction with a free-electron gas (FEG) $[12,13]$. At these intermediate and low ion energies, a series of recent experimental and theoretical studies aim on improving our understanding of the ion-solid interaction [14-16], as well as on exploring complex nonequilibrium solid-state physics [17-19].

Aside from these efforts, there exists a general need from the broad scientific community for more reliable and accurate stopping data [20]. The Nuclear Data Section of the Atomic Energy Agency (IAEA) [21] maintains a database of experimentally deduced stopping powers for light and heavy ions, together with a number of theoretical and semiempiricalbased comparisons for several ion-target combinations [22]. Although this database holds values from more than 850 references [23], a closer examination of the available data quickly reveals open issues: For many ion-solid combinations, either there are no experimental data available, or data sets from different experimental works are widely scattered, well beyond their stated uncertainties. Only a few chemical elements, that are readily available with high purity (mainly $\mathrm{C}, \mathrm{Al}, \mathrm{Si}, \mathrm{Cu}, \mathrm{Ag}$, and $\mathrm{Au}$ ), represent the most abundantly measured targets in the IAEA database [24] and correspond to more than $60 \%$ of all available data of stopping power for light ions. Data for transition and rare-earth metals, however, are rather scarce.

For both manifold technological applications and also for establishing a more complete picture of the dependence of the stopping power on the electronic structure of the material, reliable stopping data for transition and rare-earth metals are thus highly relevant. In a recent work [25], we have demonstrated that the electronic stopping of slow protons in a rareearth metal (Gd) and a transition metal (Ta) was found to be extraordinarily high when compared to other transition metals (such as Pt and $\mathrm{Au}$ ) in both low- (FEG) and high- (Bragg peak) 
energy regimes. At low velocities ( $v<0.2$ a.u.), electronic stopping of Au can be well described using a nonlinear FEG model [26] with low density FEG $\left(r_{s} \approx 3.01\right.$ a.u.) [27]. For the late transition metal Pt the density of states (DOS) is considered to be high up to the Fermi level due to the $d$ electrons; also there the same model well describes the stopping power up to the Fermi velocity [28]. On the other hand, for Gd and Ta, with only partially filled $f$ and $d$ shells, it was observed that the description of electronic stopping in a FEG model completely fails, as the experimentally deduced $r_{s}$ would lead to an unreasonably high number of valence electrons without any physical meaning. The stopping power of Gd was also measured up to the Bragg peak, and the maximum $\varepsilon$ was found to be very high $\left(\varepsilon_{\mathrm{Gd}} \approx 48 \times 10^{-15} \mathrm{eV} \mathrm{cm}{ }^{2} /\right.$ atoms at $\left.80 \mathrm{keV}\right)$, different from first-principle expectations [25]. These findings for Gd and Ta were associated with the high DOS, both below and above the Fermi level. These observations are expected to also be relevant for the magnitude of electronic stopping close to the Bragg peak. The scarcity of data combined with often limited traceability of how the data were obtained for this class of highly reactive materials is, so far, hampering a systematic analysis for early and late transition metals.

We present results from experimental studies of stopping cross sections of pure transition metals $(\mathrm{Nb}, \mathrm{Pd}, \mathrm{Ta}$, and $\mathrm{Pt})$ for protons and for helium in backscattering geometry in a wide energy range (from tens of $\mathrm{keV}$ to few $\mathrm{MeV}$ ), thus including the Bragg peak regime. These results, together with earlier studies by the authors on $\mathrm{V}, \mathrm{Ni}, \mathrm{Cu}, \mathrm{Ag}$, and $\mathrm{Au}$ allow us to compare consistent data sets for early and late transition as well as noble metals. The investigated metals feature significantly different electronic structures, i.e., partially unoccupied and filled $d$ subshells; as well as for period 6 additionally occupied $f$ states. In this light, we compare our results to other data from the IAEA database and to the most recent predictions by SRIM [29] and DPASS [30] codes and to different theories (Kaneko's [31] and CASP [32]) since we aim at understanding how different band structures might affect both position and magnitude of the stopping maximum.

\section{EXPERIMENT AND EVALUATION}

The stopping power of all metals investigated in this work was deduced from measurements in backscattering geometries carried out at four different particle accelerators to cover a broad energy range, with the surplus of cross-checking the consistency between data sets produced using several setups, geometries, and energy ranges. Specifically, low-energy measurements were performed at the AN-700 accelerator at the Johannes Kepler University (JKU) in Linz, Austria and at the 350-KV Danfysik implanter at the Uppsala University (UU) in Sweden, while medium- and high-energy measurements were made at the 2-MV NEC-5SDH tandem accelerator at the University of Sao Paulo (USP), Brazil and at the 5-MV NEC15SDH-2 tandem accelerator at Uppsala University, Sweden. Details of the experimental setups, including geometries, energy range, detector resolution, and other experimental conditions, are summarized in Table S1 in the Supplemental Material [33]. The accuracy of the impinging beam energy for all setups is confirmed to be better than $0.5 \%$. Details regarding procedures for primary beam energy calibration of each accelerator at JKU, USP, and UU (Implanter and Tandem) can be found, respectively, in Refs. [34-37].

The experimental procedure is based on the fact [38] that the height of the backscattering spectrum of a given sample contains information on the stopping power [39]. Different from previous works-where the stopping power was basically deduced directly from the spectrum height of a sample [40-42] — we have deduced the stopping data relative to reference samples with similar atomic number and well-known stopping power values (in this work: $\mathrm{Cu}, \mathrm{Ag}$, and $\mathrm{Au}$ ). As an example, the backscattering spectrum of a material of interest (e.g., Ta) is recorded relative to a reference sample (e.g., Au), and the ratio of the spectrum heights $H_{\mathrm{Ta}}^{\mathrm{Expt}} / H_{\mathrm{Au}}^{\text {Expt }}$ is compared to the ratio obtained from the simulations $H_{\mathrm{Ta}}^{\mathrm{Sim}} / H_{\mathrm{Au}}^{\mathrm{Sim}}$ using Monte Carlo TRBS [43] and SIMNRA [44] codes. These simulations allow us to account for corrections due to screening and multiple scattering - especially towards low energies-as well as for adjusting the stopping power used as input $[45,46]$. The electronic stopping power of the sample of interest can thus be calculated as

$$
\left[\varepsilon_{\mathrm{Ta}}^{\mathrm{Expt}}\right]=\sum_{E \min }^{E \max }\left(\frac{H_{\mathrm{Au}}^{\mathrm{Expt}} / H_{\mathrm{Au}}^{\mathrm{Sim}}}{H_{\mathrm{Ta}}^{\mathrm{Expt}} / H_{\mathrm{Ta}}^{\mathrm{Sim}}}\right)\left[\varepsilon_{\mathrm{Au}}^{\mathrm{Expt}}\right] \frac{\left[\varepsilon_{\mathrm{Ta}}^{\mathrm{Sim}}\right]}{\left[\varepsilon_{\mathrm{Au}}^{\mathrm{Sim}}\right]},
$$

where $[\varepsilon]$ is the stopping cross section factor $[\varepsilon]=$ $\left[K \varepsilon\left(E_{0}\right) / \cos (\alpha)+\varepsilon\left(K E_{0}\right) / \cos (\beta)\right]$ (with $K$ being the kinematic factor, and $\alpha$ and $\beta$ the incident and exit angle with respect to the beam direction and sample normal, respectively), evaluated in a certain energy range $\left(E_{\min }\right.$ and $\left.E_{\max }\right)$ slightly below the high-energy edge of the spectra. For a more detailed description of the present procedure, we refer to Ref. [47].

As reference stopping data, we chose the noble metals $\mathrm{Cu}, \mathrm{Ag}$, and $\mathrm{Au}$ due to their vicinity in atomic number to our samples of interest, minimizing potential uncertainties from scattering potentials, and exploiting the abundancy of well-established data sets in the literature [48,49]. We have selected in total 1368 values from IAEA ( 856 for $\mathrm{H}$ and 512 for He; see the list of references in Table S2 in the Supplemental Material [33]). Most of the data sets feature absolute measurements, present well-established traceability of their uncertainties [50], and are in full agreement with various reliability and statistical studies [51,52] performed in a series of works by Paul and Schinn $[53,54]$. Overall accuracy of the reference stopping data used in this work is found to be $\approx 2 \%$ averaged over the entire energy chain of the present measurements (for more discussions we refer to [47]).

In Fig. 1(a) we show typical spectra obtained in relative backscattering measurement on reference $\mathrm{Au}$ (black circles) and $\mathrm{Ta}$ (red circles), using $200-\mathrm{keV} \mathrm{D}^{+}$ions as the primary beam and the detector placed at $\theta=154.6^{\circ}$ scattering angle with a resolution of $\approx 7 \mathrm{keV}$ full width at half maximum (FWHM) for the entire amplification chain-see Table S1 [33] for more details). In the energy interval used for the evaluation [vertical blue dashed lines, panel (a)], both simulation and experiment perfectly coincide. To show the sensitivity of this procedure [i.e., Eq. (1)] to the plateau height of the Ta simulated spectrum, the final $[\varepsilon]$ is changed by $\pm 10 \%$ for comparison (red dashed lines). It is essential in this procedure to ensure the same collected charge per spectrum, and this is achieved by repeated alternating measurements of 

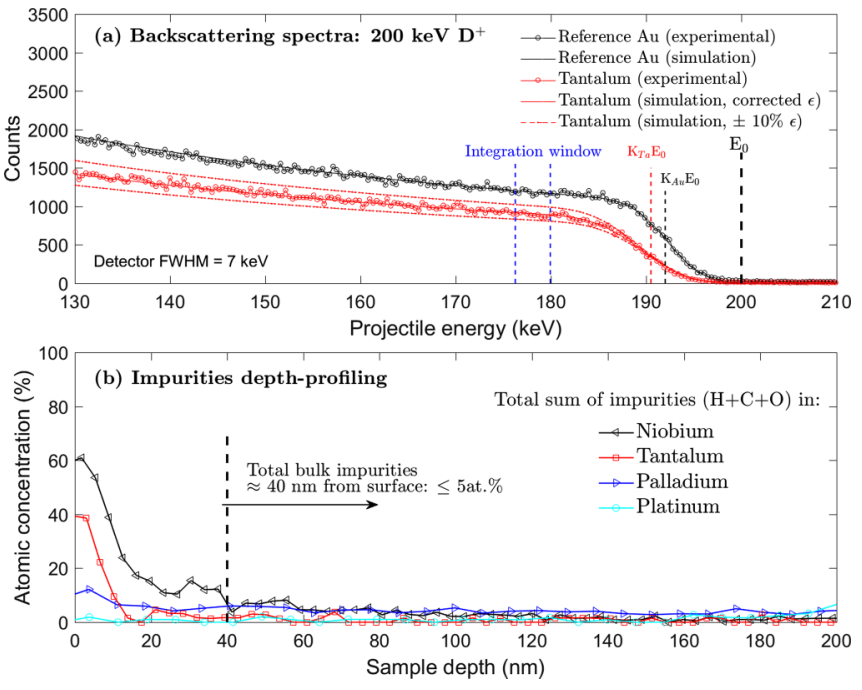

FIG. 1. (a) Experimental (open circles) and simulated (solid lines) energy spectra of $200-\mathrm{keV} \mathrm{D}^{+}$ions backscattered from $\mathrm{Au}$ (black data) and Ta (red data). Simulation using $[\varepsilon]$ deduced from Eq. (1) perfectly agrees with the experiment in the energy interval used for the evaluation (dashed blue lines). For comparison, the $\varepsilon$ value is changed by $\pm 10 \%$. (b) Total depth profile of the summed impurity $(\mathrm{H}+\mathrm{C}+\mathrm{O})$ from each sample investigated. The vertical dashed line indicates a depth region in the bulk in which the contaminants are found in levels $\leqslant 5$ at. $\%$.

reference and interest samples. In this work, the final statistical uncertainty arising from the total collected charge was, on average, $\approx 1 \%-2 \%$, depending on the energy and particle accelerator used (for more details of the charge collection procedure, see [47]).

As transition metals are commonly rather chemically reactive, knowledge on the purity of the samples is crucial to not affect the accuracy of deduced stopping results. All samples employed in this work are commercially available polycrystalline metallic bulks $(\approx 10 \times 10 \mathrm{~mm})$, with nominal purity of at least $99.5 \%$, and thickness ranging from 0.1 to 1 $\mathrm{mm}$. Their level of impurities was routinely checked by means of coincidence time-of-flight elastic recoil detection analysis (ToF-ERDA) at Uppsala University (see Refs. [55,56] for setup details and data analysis, respectively). Only small amounts of $\mathrm{H}, \mathrm{C}$, and $\mathrm{O}$ were found in (and on) the samples (quantification limit estimated to be $\approx 0.1$ at. $\%$ for the employed ToF-ERDA analysis). In Fig. 1(b), example depth profiles of impurities of each transition metal are presented (i.e., the sum of the $\mathrm{H}, \mathrm{C}$, and $\mathrm{O}$ amounts for convenience). The highest concentration level of impurities is located close to the surface, within $\approx 40 \mathrm{~nm}$ (dashed line), while in the bulk of the metals the purity is found to be always better than $\sim 95$ at.\%. Near to the surface, oxygen (not shown) is the major element present aside from the metal, likely from metal oxide layers, predominantly for the more reactive early transition metals [compare, e.g., $\mathrm{Nb}$ to $\mathrm{Pt}$ in panel (b)]. The influence of impurities has been considered in the evaluation procedure by employing Bragg's rule [57]. Such correction has been demonstrated to be adequate for low- $Z$ impurities in the present energy range [58].

\section{RESULTS AND DISCUSSIONS}

Electronic stopping cross sections of $\mathrm{Nb}, \mathrm{Pd}$, Ta, and $\mathrm{Pt}$ deduced for protons (and deuterons) are presented in Figs. 2(a)2(d), respectively. Data sets indicate the corresponding setups: Blue left and right triangles are data for $\mathrm{D}^{+}$and $\mathrm{H}^{+}$, respectively, from JKU, Linz; blue stars are data from UU (using the implanter), Uppsala; blue diamonds are data from USP, Sao Paulo; and, finally, blue squares are results from UU (using the Tandem accelerator), Uppsala. The respective energy range of each data set is summarized in Table S1 [33]. Excellent agreement between data sets from all laboratories (i.e., different setups, geometries, and energy ranges) is observed, indicating consistency of the experimental and data evaluation procedures. The overall accuracy of the experimental data is found to be $\approx 3 \%$ on average, considering random and systematic uncertainties, following criteria in [59]. A more detailed description of the budget of uncertainties for this present approach can be found in Sec. 5 of [47]. The experimental data are available in tabular form in the Supplemental Material [33] (see Tables S3 and S4 for protons and helium, respectively). To extract a smooth and continuous behavior of $\varepsilon$ over the entire measured energy range, a fitted interpolation of the obtained values for each metal is obtained using the Ziegler-Biersack (ZB) parametrization [60] (blue continuous line). The averaged precision of the $\mathrm{ZB}$ fit to the experimental data (i.e., the spread of the data around the fitted line) is found to be within $\pm 1.5 \%$.

The results shown in Fig. 2 are also compared to data from the literature (black open circles) using the IAEA compilation [22]. For high energies (i.e., $E \geqslant 800 \mathrm{keV}$ ), in general, very good agreement between our data and the literature is observed for all the samples, except for only one data set of protons in Ta [panel (c)] at $E \geqslant 700$, which is found systematically low. For Pd (panel b), for instance, our data agree with recent data [61] at energies $\geqslant 300 \mathrm{keV}$ (black solid circles). Towards lower energies - particularly around the maximum $\varepsilon$ region (i.e., the Bragg peak around $100 \mathrm{keV}$ )—our results are found to be systematically high compared to available data for $\mathrm{Nb}$ by $\sim 3 \%$ [panel (a)], for Ta by $\sim 12 \%$ [panel (c)], and for Pt by $\sim 15 \%$ [panel (d)], and systematically low for Pd by $\sim 12 \%$ [panel (b)]. However, in this energy regime and at lower energies our data and the corresponding ZB fit are in full agreement with recently published data for Ta [25] [black solid circles, panel (c)], and for Pt [28,62,63] [black solid symbols, (d)].

Our results in Fig. 2 are also compared to the latest version of the SRIM (2013) [29] (black dashed line) and to the version 21.06 of DPASS (2020) [30] (black dot-dashed lines) codes. SRIM predictions are in good agreement with our data for all the transition metals only at high energies $(E \geqslant 1 \mathrm{MeV})$, while towards low energies $(E<1 \mathrm{MeV})$, SRIM reproduces the literature data trend. As SRIM is a parametrization method of available experimental data, guided in part by theory (ZB-like approach for energies below $1 \mathrm{MeV} / \mathrm{u}$ and a Bethe-Bloch-like approach for energies higher than $1 \mathrm{MeV} / \mathrm{u}$ ) [64], it is evident that the accuracy of SRIM prediction strongly depends on the amount and quality of the available experimental data. Deviations of up to $\sim 12 \%-15 \%$ are found between our data and SRIM outputs for, e.g., Pd, Ta, and Pt at the Bragg peak. SRIM 

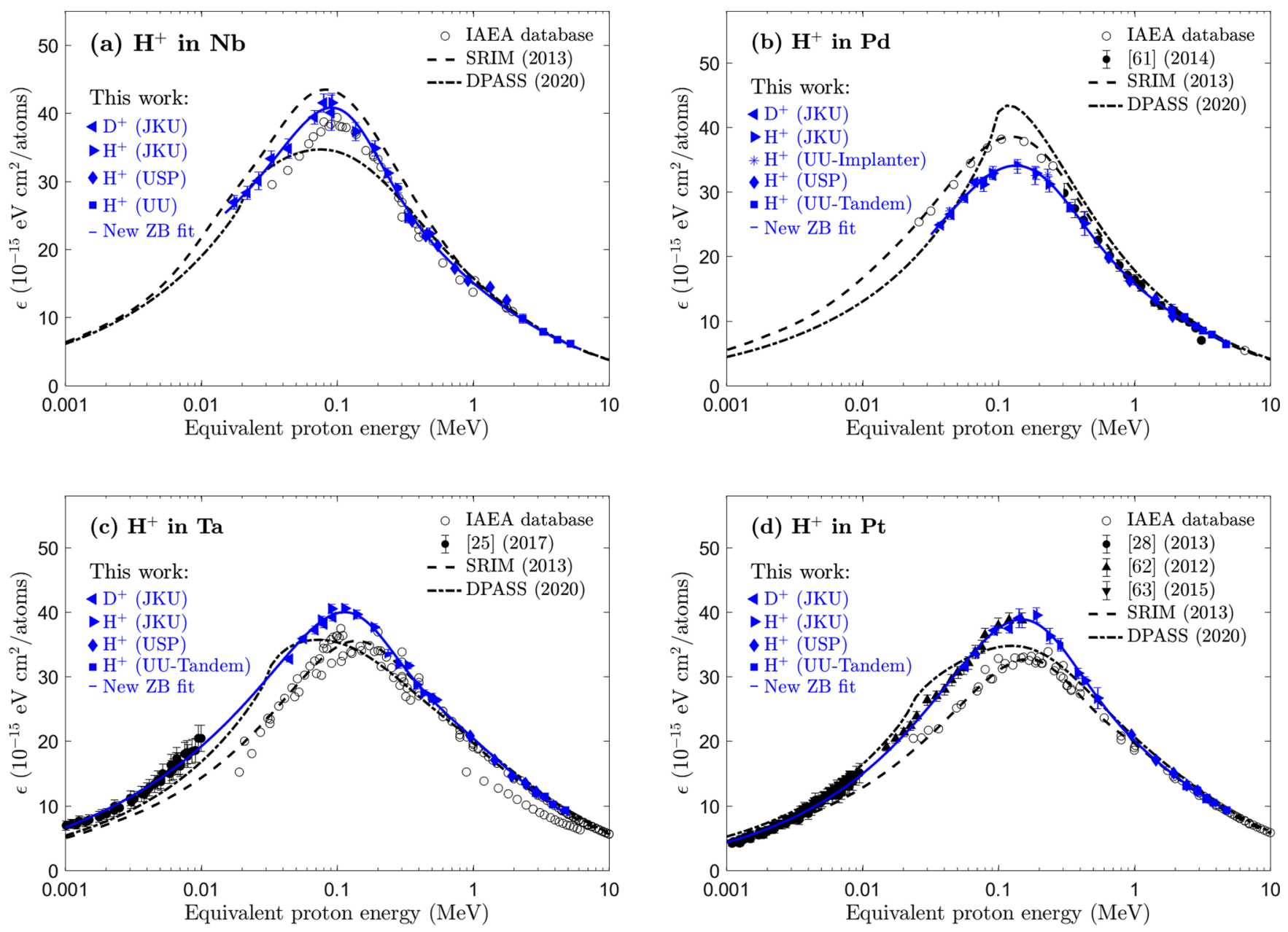

FIG. 2. Experimental stopping cross section data for $\mathrm{Nb}$ (a), $\mathrm{Pd}(\mathrm{b}), \mathrm{Ta}$ (c), and $\mathrm{Pt}$ (d) as a function of the equivalent proton energy deduced from relative backscattering measurements carried out in four different laboratories: JKU, Linz (blue solid left and right triangles); USP, Sao Paulo (blue solid diamonds); and UU, Uppsala (blue stars at Implanter and blue solid squares at Tandem). Black open and solid symbols are published values from the literature [22]. Outputs from SRIM (2013) and DPASS (2020) are shown for comparison (black dashed and dot-dashed lines, respectively). Blue continuous line represents the ZB fit using our data sets (see text for details).

has the advantage, on the other hand, to be self-refined in time by being fed with more (accurate) experimental data. DPASS results are in good agreement with our data (and with the literature) for high energies $(E \geqslant 1000 \mathrm{keV})$ for all transition metals, whereas around the maximum stopping, DPASS results in lower $\varepsilon$ values compared to any experimental data for $\mathrm{Nb}$, $\mathrm{Ta}$, and $\mathrm{Pt}$ (up to $\sim 12 \%-22 \%$ ), and higher for $\mathrm{Pd}(\sim 20 \%$ ).

In Fig. 3, the stopping cross sections of $\mathrm{Nb}, \mathrm{Pd}$, Ta, and $\mathrm{Pt}$ for helium ions are presented as a function of energy, respectively, in panels $(\mathrm{a}-\mathrm{d})$. Again, excellent agreement between data sets obtained in different setups (blue filled symbols) is observed. The final averaged accuracy of the data for helium is found to be $\approx 3 \%$ (see Sec. 5 in [47] for further discussions on the deduced uncertainties). The $\mathrm{ZB}$ fit over the whole measured energy chain is also shown for comparison in Fig. 3 (blue continuous line, average precision $\pm 1.5 \%$ ). For $\mathrm{Pd}$ and Ta [panels (b) and (c), respectively], our data agree very well with literature stopping values around (and above) the Bragg peak, while a clear disagreement between this work and literature data is observed at energies $\leqslant 400 \mathrm{keV}$. For Pt [panel (d)], our data coincide with published data around and above the
Bragg peak. Towards low energy (i.e., $E<1 \mathrm{MeV}$ ), our Pt data agree only with recent (absolute) measurements evaluating the width of spectra obtained in medium energy ion scattering [62] [black solid triangles, panel (d)]. Additionally, our ZB fit for Pt coincides with also recent low-energy stopping data deduced from the spectrum width in low-energy ion scattering [28] (black solid circles).

SRIM predictions (black dashed line) for helium stopping data agree with our data at energies $E \geqslant 1 \mathrm{MeV}$ for Pd, Ta, and $\mathrm{Pt}$ [panels $(\mathrm{b}-\mathrm{d})$, respectively], whereas for $\mathrm{Nb}$, an evident disagreement is observed $(\sim 10 \%)$, mainly due to the lack of available data-SRIM most likely adopts interpolations using data from neighboring elements. As the accuracy of SRIM for $\mathrm{Nb}$ stopping data using helium as a projectile is limited, further comparisons to our data are, hence, meaningless. Around the Bragg peak (i.e., at energies around $1 \mathrm{MeV}$ ), SRIM agrees to data from this work for Pd $(\sim 2 \%)$, and it is slightly worse for $\mathrm{Ta}$ and $\mathrm{Pt}(\sim 5 \%)$. On the other hand, towards lower energies $(E<1 \mathrm{MeV})$ a more prominent disagreement of SRIM is rather evident for $\mathrm{Pd}, \mathrm{Pt}$, and Ta. Predictions by DPASS (black dot-dashed line) are in accordance with our 

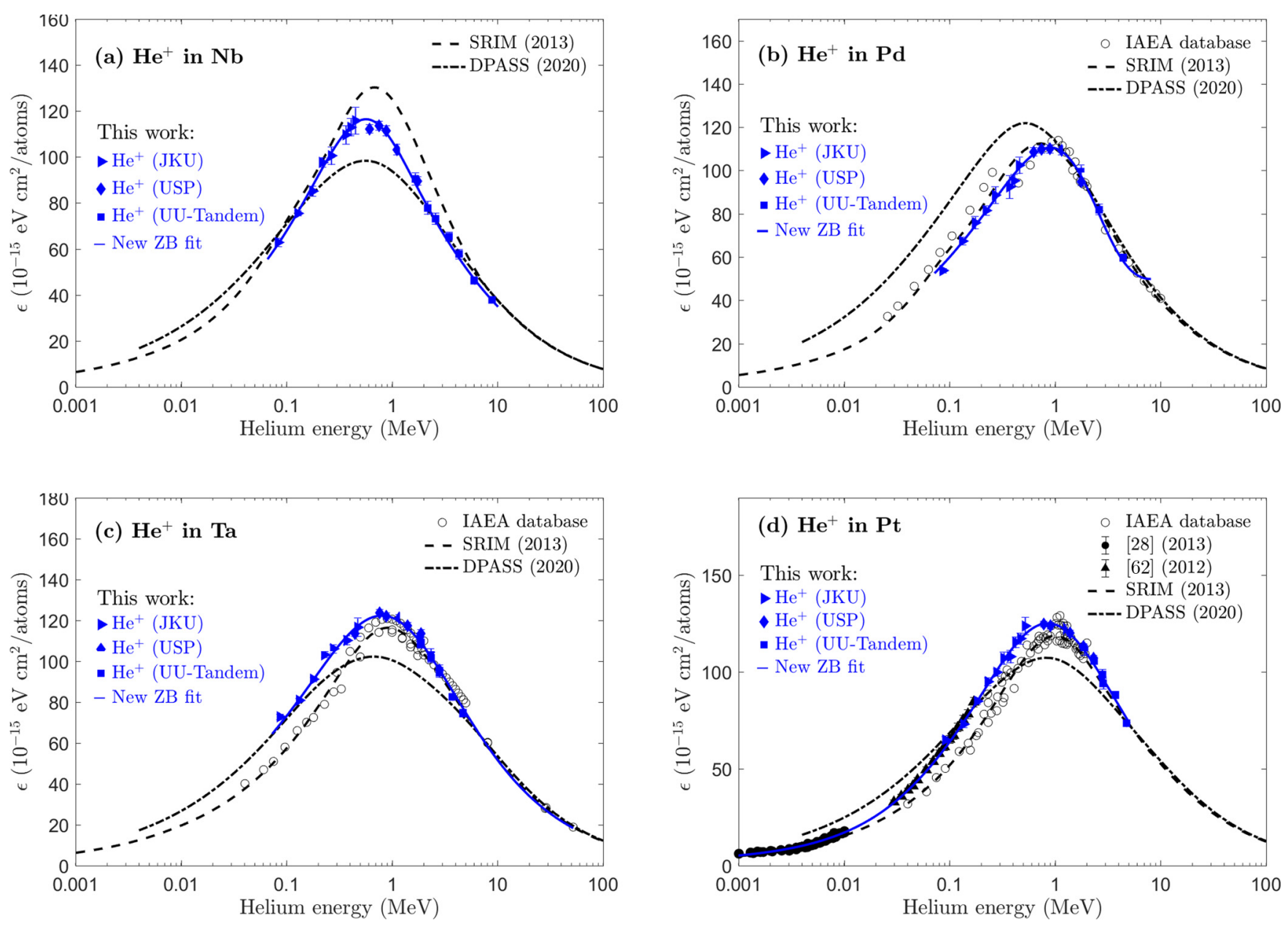

FIG. 3. Experimental stopping cross section of $\mathrm{Nb}$ (a), $\mathrm{Pd}$ (b), Ta (c), and Pt (d) as a function of the helium energy. Measurements for helium are carried out in three different laboratories (see respective blue solid symbols). Black open circles are published data from the literature. Other black solid symbols are more recent data from the literature. SRIM (2013) and DPASS (2020) predictions are shown as dashed and dot-dashed lines, respectively. Blue continuous lines represent the ZB fit over the entire energy range of the measured data (see text for details).

experimental data for high energies $(E>1 \mathrm{MeV})$, whereas at the Bragg peak they are found to be systematically lower than our data Fine with us values for $\mathrm{Nb}, \mathrm{Ta}$, and $\mathrm{Pt}(\sim 9$ up to $\sim 20 \%)$, and systematically higher for $\mathrm{Pd}(\sim 10 \%)$. Shape and position of the experimentally observed Bragg peak is generally better reproduced for helium ions than for protons.

In the following, we discuss observable systematics with respect to the Bragg peak by comparing the stopping maximum and the position of several transition metals (this work and the literature) as a function of the sample atomic number $Z_{2}$. Specifically, the $\varepsilon$ values at the maximum and the corresponding energy positions for protons and helium are compared among elements within the periods 4 ( Ti, V, Cr, Ni, and $\mathrm{Cu}$ ), $5(\mathrm{Zr}, \mathrm{Nb}, \mathrm{Mo}, \mathrm{Pd}$, and $\mathrm{Ag})$, and 6 (Ta, W, Pt, and $\mathrm{Au})$. For $\mathrm{Nb}, \mathrm{Pd}, \mathrm{Ta}$, and $\mathrm{Pt}$ we used data presented in this work (i.e., Figs. 2 and 3). For the noble metals $(\mathrm{Cu}, \mathrm{Ag}$, and $\mathrm{Au}$ ), we adopted experimental data discussed in detail in Sec. 2. For V, we employed data from [47]. For $\mathrm{Ti}, \mathrm{Cr}$, and $\mathrm{Ni}$, we used values (and trends) based on recent experimental works [65-68] and intercomparisons [69]. Finally, for $\mathrm{Zr}$, Mo, and $\mathrm{W}$, we relied on the available data in IAEA that, unfortunately for these elements, are limited and show a significant scatter of up to $5 \%-10 \%$ around the Bragg peak. To avoid problems arising from data interpolation, we adopted a similar ZB fit as previously discussed for elements other than the ones presented in Figs. 2 and 3. The final averaged precision of the fit for these other elements (i.e., Ti, V, Cr, Ni, Cu, Zr, Mo, Ag, $\mathrm{W}$, and $\mathrm{Au}-$ not shown) around the Bragg peak was found to be within $\pm 1 \%-2 \%$.

Figures 4(a) and 4(b) show the comparison between the position of the maximum stopping (in $\mathrm{keV}$ ) of the transitions metals as a function of $Z_{2}$ for protons and helium, respectively. Elements from period 4 are represented by black circles, period 5 by blue triangles, and period 6 by red diamonds. Solid symbols are the fitting data using stopping values from this work and Ref. [47] (for V), whereas open symbols are the fitting data using literature values (discussed above). To guide the eye, a linear trend is adjusted among elements within the same period (solid lines). Regardless the period and the projectile, we can observe a linear increase of the maximum stopping position with increasing $Z_{2}$ [panels (a,b) for protons and helium, respectively). In panels (c,d), we depict the maximum value of $\varepsilon, \varepsilon_{\max }$, for protons and helium, respectively, as a function of $Z_{2}$ [same symbols as for panels $(\mathrm{a}, \mathrm{b})$ ]. Note that 

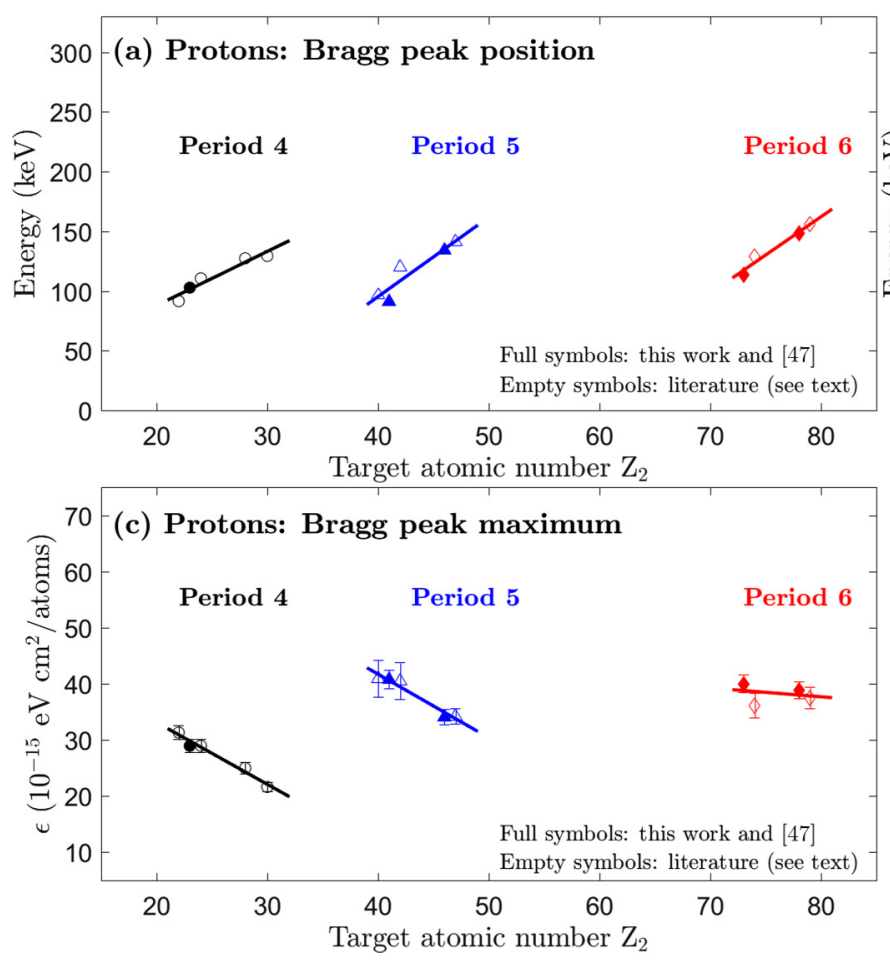
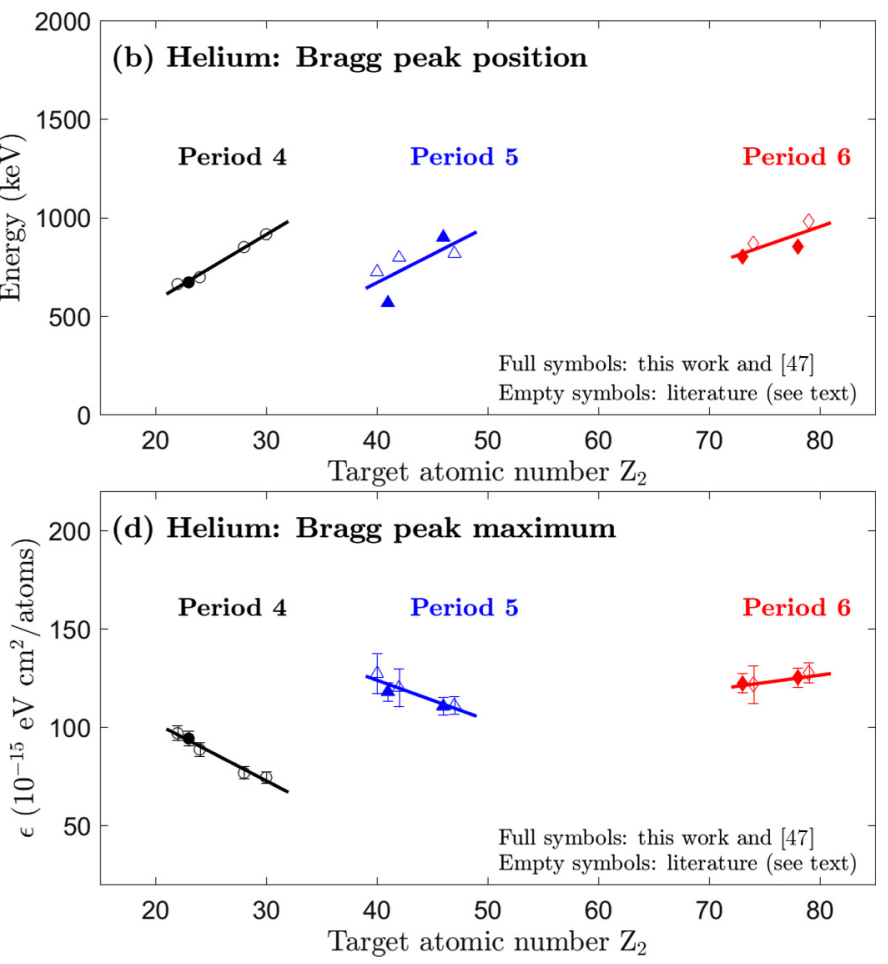

FIG. 4. Panels (a,b) show the maximum stopping position (in keV) as a function of $Z_{2}$ for 14 elements from three different periods, for protons and helium, respectively. Panels (c,d) depict the corresponding maximum stopping cross section values as a function of $Z_{2}$, for protons and helium, respectively. Period 4: Ti, V, Cr, Ni, and $\mathrm{Cu}$ (black circles); period 5: Zr, Nb, Mo, Pd, and Ag (blue triangles); and, finally, period 6: Ta, W, Pt, and $\mathrm{Au}$ (red diamonds). The solid lines are linear fits to the data.

larger error bars are attributed to some chemical elements in the literature as discussed above (i.e., Zr, Mo, and W). Similar as for the determined energy of the stopping maximum [panels (a,b)], in this limited range of $Z_{2}$ a linear trend (weighted by the uncertainties) is observed also for $\varepsilon_{\max }$, as a function of $Z_{2}$, for both protons and helium. However, while the magnitude of $\varepsilon_{\max }$ decreases throughout periods 4 and 5 , a different trend is found in period 6: for protons [panel (c)] the maximum stopping is almost constant, whereas for helium the magnitude slightly increases with increasing atomic number [panel (d)].

Attempts were made to correlate such a $Z_{2}$ dependence, the so-called $Z_{2}$ oscillations, at even higher proton energies $(18.5 \mathrm{MeV})$ with physical properties such as, e.g., density and conductivity - but without success; the electronic band structure was thus left as the most likely cause [70]. The difference of the magnitudes of $\varepsilon_{\max }$ observed in this work between elements from periods 4-6 can indeed be associated with their different band structures. Periods 4 and 5 feature transition metals with partially and fully occupied $d$ states in the valence band, whereas elements from period 6 feature additionally fully occupied $4 f$ shell electrons at energies below the conduction band. For $\mathrm{Gd}$ the valence band is partially filled with $f$ electrons, and for protons $\varepsilon_{\max }$ was found surprisingly high, $\left(\varepsilon_{\max } \approx 48 \times 10^{-15} \mathrm{eV} \mathrm{cm}^{2} /\right.$ atoms $)$ [25], i.e., even higher than for proton stopping in period 6 $\left(\varepsilon_{\max } \approx 38 \times 10^{-15} \mathrm{eV} \mathrm{cm}^{2} /\right.$ atoms) investigated in this work. Obviously, the $f$ electrons contribute considerably to electronic stopping of protons up to the Bragg maximum not only in rare-earth metals [25], but also in transition metals in period
6. The subtly different $Z_{2}$ dependence of $\varepsilon_{\max }$ in elements in period 6 for protons and helium [compare red lines in Figs. 4(c) and 4(d)] may be related to effects beyond excitation of target electrons by a point charge, such as charge-exchange effects in a more complex projectile like He.

In Fig. 5 we compare the experimentally deduced stopping cross section with three different theoretical approaches, for 200-keV protons [panel (a)] and for 1000-keV helium [panel (b)]. In the first approach, stopping power calculations by Kaneko [31,71] (black dashed line) rely on Lindhard's theory of stopping power for charged particles in a free-electron gas [72]. In a different approach, the DPASS program (red dot-dashed line) provides stopping power based on binary theory [73], as an extension of Bohr's classical approach [74], but with improved modeling (see [75] and references therein). Finally, predictions from the CASP code [32] are also shown (blue continuous line), consisting of stopping powers deduced according to the unitary convolution approximation (UCA) [76] for energies in the Bethe regime and to the transport cross section (TCS) approach [77] for ion velocities in the FEG regime. The energies 200 and $1000 \mathrm{keV}$ were chosen for protons and helium, respectively, since there Kaneko's results are available [31], and they are sufficiently close to the energies of the stopping maximum.

As one can see in Fig. 5, at these energies, all three theoretical models agree mutually within $\pm 10 \%-15 \%$ on average up to $Z_{2} \leqslant 50$ for protons and $Z_{2} \leqslant 30$ for helium and show similar trends in their $Z_{2}$ dependence. At higher $Z_{2}$, values, an accentuated discrepancy is observed between CASP and DPASS 


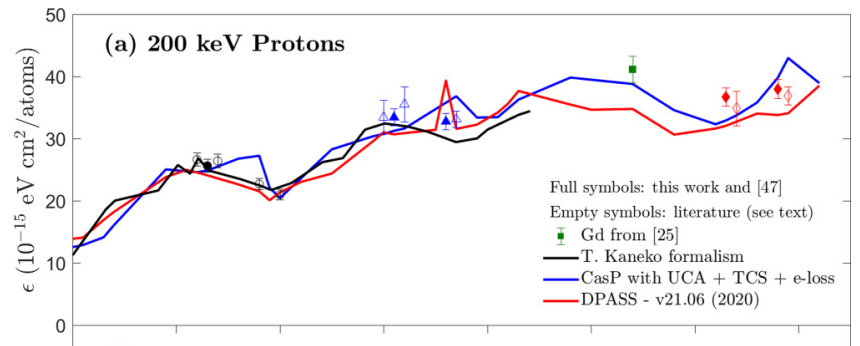

(b) $1000 \mathrm{keV}$ Helium

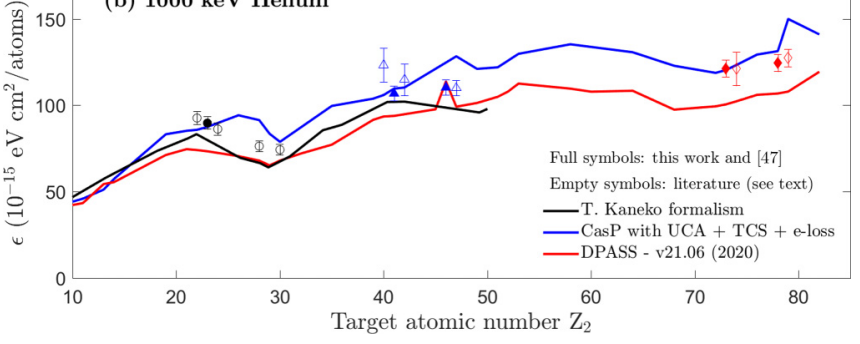

FIG. 5. Experimental (solid symbols from this work and from Refs. [25,47] and open ones from the literature) and theoretical (solid lines) stopping cross section $\varepsilon$ as a function of the target atomic number $Z_{2}$ for $200-\mathrm{keV}$ protons (a) and for $1000-\mathrm{keV}$ helium (b). Experimental data represent transition metals from period $4(\mathrm{Ti}, \mathrm{V}$, $\mathrm{Cr}, \mathrm{Ni}$, and $\mathrm{Cu}$-black circles), 5 ( $\mathrm{Zr}, \mathrm{Nb}, \mathrm{Mo}, \mathrm{Pd}$, and $\mathrm{Ag}$-blue triangles) and 6 (Ta, W, Pt, and $\mathrm{Au}-$ red diamonds). Theories are Kaneko's formalism, CASP, and DPASS (see text for details).

(Kaneko's formalism ends at $Z_{2}=54$ and 50 for protons and helium, respectively). When compared to experimental data (black circles for period 4, blue triangles for period 5, and red diamonds for period 6), all the theoretical approaches yield a better agreement of experimental data from periods 4 and 5, particularly for protons-e.g., Kaneko's approach reproduces with good accuracy both $\varepsilon$ and $Z_{2}$ oscillations for elements from period 4 . For elements from period 6 , there is an evident discrepancy between CASP and DPASS: Although both show $Z_{2}$ oscillations, CASP yields generally a higher stopping cross section, which in turn, on average better reproduces the experimental data. For comparison, the stopping cross section of $\mathrm{Gd}$ for 200-keV protons from [25] has also been included in panel (a) (green solid square) evidencing that only CASP is compatible to reproduce its stopping power reasonably well. For CASP calculations, we activate expert options to compute the stopping contribution from the target inner shells (via UCA) and contributions from the valence electrons (via TCS), as well as projectile excitation ( $e$ loss). As a result, CASP reproduces experimental data for chemical elements from the sixth period more satisfactorily than DPASS, at least for these energies and in particular for helium projectiles. Note that, in this context, DPASS also requires input in the form of empirical data (e.g., mean excitation energy $I$ and shell binding energies $U)$.

\section{CONCLUSIONS}

Experimental electronic stopping cross sections of different transition metals $(\mathrm{Nb}, \mathrm{Pd}, \mathrm{Ta}$, and $\mathrm{Pt}$ ) have been deduced from relative backscattering measurements for a wide energy range of protons $(\approx 50-5000 \mathrm{keV})$ and helium $(\approx 80-10000 \mathrm{keV})$. The measurements were carried out using different setups, geometries, and energy range from four different laboratories: Linz, Austria; Sao Paulo, Brazil; and Uppsala, Sweden. The stopping data are obtained with an average uncertainty of $\approx 3 \%$ for protons and helium, in the whole energy interval. Agreement between the different data sets is excellent, showing consistency of both experimental and evaluation approaches. As the present methodology relies on ratios of spectrum heights obtained using bulk samples, it leads to a better control over sample purity, thus overcoming several potential problems such as, e.g., surface and bulk contaminations that affect the accuracy of the evaluated stopping power data. In the entire energy interval of interest, a Ziegler-Biersack type function is fitted to the experimental data to extract a smooth and continuous stopping power curve for both projectiles, within final averaged precision better than $2 \%$.

Our experimental results (available also in tabular form in the Supplemental Material [33]-Tables S3 for protons and S4 for helium) cover a wide energy range including the maximum stopping region (i.e., the Bragg peak), and represent considerable improvement in availability of accurate stopping data for transition metals. For example, our data represent a data set of helium stopping in $\mathrm{Nb}$. Our data are compared with available literature data, with semiempirical predictions from SRIM (2013) and with theoretical calculation from DPASS (2020), with the results being different from the literature and SRIM by up to $\sim 5 \%-10 \%$ for protons and up to $\sim 15 \%$ for helium, as well as from DPASS of up to $\sim 20 \%$ for protons and helium.

We also presented an experimental study about systematics of the stopping power maximum, i.e., its maximum value $\varepsilon_{\max }$ and its energy position, as a function of target atomic number $Z_{2}$ for 14 different transition metals from three distinct periods. We demonstrate that there is a common trend for the energy maximum position of $\varepsilon_{\max }$, which-in the narrow range of $Z_{2}$ investigated-increases linearly with increasing $Z_{2}$ among elements in the same period regardless of the projectile. By comparing the maximum stopping value, on the other hand, a similar trend is only verified for periods 3 and 4 , with decreasing $\varepsilon_{\max }$ values with increasing $Z_{2}$, for protons and helium. For period 6 , however, the stopping maximum as a function of $Z_{2}$ is almost constant for protons and increases slightly for helium with increasing $Z_{2}$. This behavior is attributed to the fully occupied $4 f$ shells of the transition metals from period 6 , which contribute to electronic stopping and cause the different properties of the Bragg peaks in period 6 transition metals compared to periods 4 and 5. This evidence is also observed when these elements are compared against full theoretical models, in which the CASP code (with transport cross section approach for the FEG regime) was demonstrated to be the closest theoretical approach to predict our results for both protons and helium around the Bragg peak regime.

\section{ACKNOWLEDGMENTS}

The authors are thankful to Professor Manfredo Tabacniks (USP, Sao Paulo) for fruitful discussions in the early stages of this work and for granting access to the laboratory. The 
authors would like also to acknowledge M. A. Rodrigues and J. Åström for the support of accelerator operation in Brazil and Sweden, respectively. Financial support from VR-RFI
(Contracts No. 821-2012-5144 and No. 2017-00646_9) and the Swedish Foundation for Strategic Research (SSF, Contract No. RIF14-0053) is gratefully acknowledged.
[1] P. Sigmund, Particle Penetration and Radiation Effects, Vol. 2 (Springer, Cham, Switzerland, 2014).

[2] P. Sigmund, Stopping power in perspective, Nucl. Instrum. Methods Phys. Res., Sect. B 135, 1 (1998).

[3] M. Curie and P. Curie, Sur la charge électrique des rayons déviables du radium, C. R. Acad. Sci. 130, 647 (1900).

[4] S. Creutzburg, E. Schmidt, P. Kutza, R. Loetzsch, I. Uschmann, A. Undisz, M. Rettenmayr, F. Gala, G. Zollo, A. Boulle, A. Debelle, and E. Wendler, Defects and mechanical properties in weakly damaged Si ion implanted GaAs, Phys Rev. B 99, 245205 (2019).

[5] J. Jeynes and J. L. Colaux, Thin film depth profiling by ion beam analysis, Analyst 141, 5944 (2016).

[6] M. Mayer, S. Möller, and M. Rubel et al., Ion beam analysis of fusion plasma-facing materials and components: Facilities and research challenges, Nucl. Fusion 60, 025001 (2020).

[7] B. He, X. J. Meng, and Z. G. Wang et al., Ab initio research of energy loss for energetic protons in solid-density Be, Phys. Plasmas 24, 033110 (2017).

[8] M. E. Alcocer-Ávila, M. A. Quinto, and J. M. Monti et al., Proton transport modeling in a realistic biological environment by using TILDA-V, Sci. Rep. 9, 14030 (2019).

[9] P. de Vera, R. Garcia-Molina, and I. Abril, Simulation of the energy spectra of swift light ion beams after traversing cylindrical targets: A consistent interpretation of experimental data relevant for hadron therapy, Eur. Phys. J. D 73, 209 (2019).

[10] H. Bethe and J. Ashkin, in Experimental Nuclear Physics, edited by E. Segré (J. Wiley, New York, 1953), p. 253.

[11] P. Sigmund and A. Schinner, Notes on Barkas-Andersen effect, Eur. Phys. J. D 68, 318 (2014).

[12] E. Fermi and E. Teller, The capture of negative mesotrons in matter, Phys. Rev. 72, 399 (1947).

[13] A. Mann and W. Brandt, Material dependence of low-velocity stopping powers, Phys. Rev. B 24, 4999 (1981).

[14] M. Mery, L. Chen, and J. E. Valdés et al., On the determination of stopping cross-sections in ion scattering in solids and deviations from standard models, Radiat. Eff. Defects Solids 175, 160 (2020).

[15] F. Matias, P. L. Grande, M. Vos, P. Koval, N. E. Koval, and N. R. Arista, Nonlinear stopping effects of slow ion in a no-freeelectron system: Titanium nitride, Phys. Rev. A 100, 030701(R) (2019).

[16] C. C. Montanari and J. E. Miraglia, Low- and intermediateenergy stopping power of protons and antiprotons in solid targets, Phys. Rev. A 96, 012707 (2017).

[17] S. Lohmann and D. Primetzhofer, Disparate Energy Scaling of Trajectory-Dependent Electronic Excitations for Slow Protons and He Ions, Phys. Rev. Lett. 124, 096601 (2020).

[18] A. E. Sand, R. Ullah, and A. A. Correa, Heavy ion ranges from first-principle electron dynamics, Comput. Mater. 5, 43 (2019).

[19] A. Tamm, M. Caro, A. Caro, G. Samolyuk, M. Klintenberg, and A. A. Correa, Langevin Dynamics with Spatial Correlations as a Model for Electron-Phonon Coupling, Phys. Rev. Lett. 120, 185501 (2018).
[20] M. V. Moro, T. F. Silva, A. Mangiarrotti, Z. O. GuimarãesFilho, M. A. Rizzutto, and M. H. Tabacniks, Traceable stopping cross sections of $\mathrm{Al}$ and Mo elemental targets for $0.9-3.6 \mathrm{MeV}$ protons, Phys. Rev. A 93, 022704 (2016).

[21] Nuclear Data Service of the International Atomic Energy Agency, available at https://www-nds.iaea.org/.

[22] Stopping Power Database, available at https://www-nds.iaea. org/stopping/. All the data sets used in this work were first downloaded in July, 2016, and updated accordingly.

[23] C. C. Montanari and P. Dimitriou, The IAEA stopping power database, following trends in stopping power of ions in matter, Nucl. Instrum. Methods, Phys. Res., Sect. B 408, 50 (2017).

[24] K. Wittmaack, Misconceptions impairing the validity of the stopping power tables in the SRIM library and suggestions for doing better in the future, Nucl. Instrum. Methods Phys. Res., Sect. B 380, 57 (2016).

[25] D. Roth, B. Bruckner, M. V. Moro, S. Gruber, D. Goebl, J. I. Juaristi, M. Alducin, R. Steinberger, J. Duchoslav, D. Primetzhofer, and P. Bauer, Electronic Stopping of Slow Protons in Transition and Rare Earth Metals: Breakdown of the Free Electron Gas Concept, Phys. Rev. Lett. 118, 103401 (2017).

[26] P. M. Echenique, R. M. Nieminen, and R. H. Ritchie, Density functional calculation of stopping power of an electron gas for slow ions, Solid State Commun. 37, 779 (1981).

[27] D. Goebl, W. Roessler, D. Roth, and P. Bauer, Influence of the excitation threshold of $d$ electrons on electronic stopping of slow light ions, Phys. Rev. A 90, 042706 (2014).

[28] D. Goebl, D. Roth, and P. Bauer, Role of $d$ electrons in electronic stopping of slow light ions, Phys. Rev. A 87, 062903 (2013).

[29] J. F. Ziegler, SRIM code v. 2013, available at http://www.srim. org/. Downloaded on July 2017.

[30] A. Schinner and P. Sigmund, DPASS code v. 21.06, https://www. sdu.dk/DPASS. Downloaded on May 2020.

[31] T. Kaneko, $Z_{2}$ dependence of the stopping power and the effective charge for MeV helium-ion beams, Phys. Rev. A 30, 1714 (1984).

[32] P. L. Grande and G. Schiwietz, Impact-parameter dependence of the electronic energy loss of fast ions, Phys. Rev. A 58, 3796 (1998).

[33] See Supplemental Material at http://link.aps.org/supplemental/ 10.1103/PhysRevA.102.022808 for more detailed information on the experimental procedure as well as experimental stopping power data.

[34] D. Primetzhofer and P. Bauer, Trace element quantification in high-resolution Rutherford backscattering spectrometry, Nucl. Instrum. Methods Phys. Res., Sect. B 269, 1284 (2011).

[35] T. F. Silva, M. V. Moro, and G. F. Trindade et al., Ion beam analysis of $a-\mathrm{C}: \mathrm{H}$ films on alloy steel substrate, Thin Solid Films 545, 171 (2013).

[36] M. K. Linnarsson, A. Hallén, and J. Åström et al., New beam line for time-of-flight medium energy ion scattering with large 
area position sensitive detector, Rev. Sci. Instrum. 83, 095107 (2012).

[37] V. Paneta, M. Kokkoris, and A. Lagoyannis et al., Accurate accelerator energy calibration using selected resonances in proton elastic scattering and in $(p, \gamma)$ and $\left(p, p^{\prime} \gamma\right)$ reactions, Nucl. Instrum. Methods Phys. Res., Sect. B 406, 108 (2017).

[38] W. K. Chu, J. M. Mayer, and M. A. Nicolet, Backscattering Spectrometry, 1st ed. (Academic Press, San Diego, CA, 1978).

[39] J. Tesmer and M. Nastasi, Handbook of Modern Ion Beam Materials Analysis, 1st ed. (Materials Research Society, Warrendale, PA, 1995).

[40] Sh. Z. Izmailov, E. I. Sirotinin, and A. F. Tulinov, Energy loss of protons in $\mathrm{Si}, \mathrm{Ge}$ and Mo, Nucl. Instrum. Methods 168, 81 (1980).

[41] V. Ya. Chumanov, Sh. Z. Izmailov, and G. P. Pokhil et al., On the determination of energy losses by charged particles from the backscattering energy spectra, Phys. Status Solidi A 53, 51 (1979).

[42] F. G. Neshev, A. A. Puzanov, and E. I. Sirotinin et al., The determination of energy losses of nitrogen ions from the backscattering spectra, Radiat. Eff. 25, 271 (1975).

[43] J. P. Biersack, E. Steinbauer, and P. Bauer, A particularly fast TRIM version for ion backscattering and high energy ion implantation, Nucl. Instrum. Methods Phys. Res., Sect. B 61, 77 (1991).

[44] M. Mayer, SIMNRA, a simulation program for the analysis of NRA, RBS and ERDA, in The Fifteenth International Conference on the Application of Accelerators in Research and Industry, edited by J. L. Duggan, B. Stippec, and Ira Lon Morgan, AIP Conf. Proc. No. 475 (AIP, New York, 1999), p. 541.

[45] S. N. Markin, D. Primetzhofer, M. Spitz, and P. Bauer, Electronic stopping of low-energy $\mathrm{H}$ and $\mathrm{He}$ in $\mathrm{Cu}$ and $\mathrm{Au}$ investigated by time-of-flight low-energy ion scattering, Phys. Rev. B 80, 205105 (2009).

[46] D. Goebl, K. Khalal-Kouache, D. Roth, E. Steinbauer, and P. Bauer, Energy loss of low-energy ions in transmission and backscattering experiments, Phys. Rev. A 88, 032901 (2013).

[47] M. V. Moro, B. Bruckner, and P. L. Grande et al., Stopping cross section of vanadium for $\mathrm{H}^{+}$and $\mathrm{He}^{+}$ions in a large energy interval deduced from backscattering spectra, Nucl. Instrum. Methods Phys. Res., Sect. B 424, 43 (2018).

[48] H. Paul and D. Sánchez-Parcerisa, A critical overview of recent stopping power programs for positive ions in solid elements, Nucl. Instrum. Methods Phys. Res., Sect. B 312, 110 (2013).

[49] H. Paul, D. Semrad, and A. Seilinger, Reference stopping cross sections for hydrogen and helium ions in selected elements, Nucl. Instrum. Methods Phys. Res., Sect. B 61, 261 (1991).

[50] H. Paul, Comparing experimental stopping power data for positive ions with stopping tables, using statistical analysis, Nucl. Instrum. Methods Phys. Res., Sect. B 273, 15 (2011).

[51] H. Paul, New results about stopping power for positive ions: experiment and theory, in Applications in Accelerators in Research and Industry, Twenty-Second International Conference, AIP Conf. Proc. No. 1525 (AIP, Melville, NY, 2013), p. 295.

[52] H. Paul, Recent results in stopping power for positive ions, and some critical comments, Nucl. Instrum. Methods Phys. Res., Sect. B 268, 3421 (2010).

[53] H. Paul and A. Schinner, Judging the reliability of stopping power tables and programs for protons and alpha particles using statistical methods, Nucl. Instrum. Methods Phys. Res., Sect. B 227, 461 (2005).

[54] H. Paul and A. Schinner, An empirical approach to the stopping power of solids and gases for ions from ${ }_{3} \mathrm{Li}$ to ${ }_{18} \mathrm{Ar}$, Nucl. Instrum. Methods Phys. Res., Sect. B 195, 166 (2002).

[55] P. Ström, P. Petersson, and M. Rubel et al., A combined segmented anode gas ionization chamber and time-of-flight detector for heavy ion elastic recoil detection analysis, Rev. Sci. Instrum. 87, 103303 (2016).

[56] M. V. Moro, R. Holeňák, and L. Zendejas Medina et al., Accurate high-resolution depth profiling of magnetron sputtered transition metal alloy films containing light species: A multimethod approach, Thin Solid Films 686, 137416 (2019).

[57] W. H. Bragg and R. Kleeman, On the $\alpha$ particles of radium, and their loss of range in passing through various atoms and molecules, Philos. Mag. 10, 318 (1905).

[58] B. Bruckner, D. Roth, and D. Goebl et al., A note on extracting electronic stopping from energy spectra of backscattered slow ions applying Bragg's rule, Nucl. Instrum. Methods Phys. Res., Sect. B 423, 82 (2018).

[59] Working Group 1 of the Joint Committee for Guides in Metrology, Evaluation of measurement data-guide to the expression of uncertainty in measurement (GUM), Report No. JCGM 100:2008, 2008, https://www.bipm.org/utils/common/ documents/jcgm/JCGM_100_2008_E.pdf.

[60] J. F. Ziegler, J. P. Biersack, and U. Littmark, The Stopping and Range of Ions in Solids, 2nd ed. (Pergamon Press, New York, 2009).

[61] P. A. Miranda, A. Sepúlveda, J. R. Morales, T. Rodriguez, E. Burgos, and H. Fernández, Stopping power of palladium for protons in the energy range $0.300-3.100 \mathrm{MeV}$, Nucl. Instrum. Methods Phys. Res., Sect. B 318, 292 (2014).

[62] D. Primetzhofer, Inelastic energy loss of medium energy $\mathrm{H}$ and He ions in $\mathrm{Au}$ and Pt: Deviations from velocity proportionality, Phys. Rev. B 86, 094102 (2012).

[63] C. E. Celedón, E. A. Sánchez, L. Salazar Alarcón, J. Guimpel, A. Cortés, P. Vargas, and N. R. Arista, Band structure effects in the energy loss of low-energy protons and deuterons in thin films of Pt, Nucl. Instrum. Methods Phys. Res., Sect. B 360, 103 (2015).

[64] J. F. Ziegler, Stopping of energetic light ions in elemental matter, J. Appl. Phys. 85, 1249 (1999).

[65] T. T. Tran, L. Jablonka, B. Bruckner, S. Rund, D. Roth, M. A. Sortica, P. Bauer, Z. Zhang, and D. Primetzhofer, Electronic interaction of slow hydrogen and helium ions with nickelsilicon systems, Phys. Rev. A 100, 032705 (2019).

[66] M. Brocklebank, S. N. Dedyulin, and L. V. Goncharova, Stopping cross sections of protons in $\mathrm{Ti}, \mathrm{TiO}_{2}$ and $\mathrm{Si}$ using medium energy ion scattering, Eur. Phys. J. D 70, 248 (2016).

[67] P. K. Diwan and S. Kumar, $d E / d x$ and range of $\alpha$-radiations in $\mathrm{Al}, \mathrm{Ti}$ and Ni metallic foils, Nucl. Instrum. Methods Phys. Res., Sect. B 359, 78 (2015).

[68] W. H. Trzaska, V. Lyapin, T. Alanko, M. Mutterer, J. Raisanen, G. Tjurin, and M. Wojdyr, New approach to energy loss measurements, Nucl. Instrum. Methods Phys. Res., Sect. B 195, 147 (2002).

[69] D. C. Santry and R. D. Werner, Stopping power values of Ti, Ni, $\mathrm{Ag}$ and $\mathrm{Au}$ for He-4 ions, Nucl. Instrum. Methods Phys. Res., Sect. B 178, 531 (1980). 
[70] V. C. Burkig and K. R. Mackenzie, Stopping power of some metallic elements for 19.8-MeV protons, Phys. Rev. 106, 848 (1957).

[71] T. Kaneko, Energy loss of protons and helium passing through matter, Phys. Rev. A 33, 1602 (1986).

[72] J. Lindhard and A. Winther, K. Dan. Vidensk. Selsk., Mat.-Fys. Medd. 34, 4 (1964).

[73] P. Sigmund and A. Schinner, Binary theory for swift heavy ions, Eur. Phys. J. D. 12, 425 (2000).

[74] N. Bohr, On the theory of the decrease of velocity of moving electrified particles on passing through matter, Philos. Mag. 25, 10 (1913).
[75] A. Schinner and P. Sigmund, Expanded PASS stopping code, Nucl. Instrum. Methods Phys. Res., Sect. B 460, 19 (2019).

[76] G. Schiwietz and P. L. Grande, A unitary convolution approximation for the impact-parameter dependent electronic energy loss, Nucl. Instrum. Methods Phys. Res., Sect. B 153, 1 (1999).

[77] F. Matias, R. C. Fadanelli, P. L. Grande, N. E. Koval, R. Díez Muiño, A. G. Borisov, N. R. Arista, and G. Schiwietz, Ground- and excited-state scattering potentials for the stopping of protons in an electron gas, J. Phys. B 50, 185201 (2017). 